\title{
Direct Visualization of Plasmon Assisted photon emission from an Isolated Au nanorod with sharp tips
}

\author{
Arpan Maiti* \\ Department of Basic Science and Humanities, University of Engineering and Management, \\ Action Area- III, Kolkata, West Bengal 700156, India
}

\section{INTRODUCTION}

In recent years, noble metal nanoparticles (MNPs) have attracted tremendous interest in the area of photonics research owing to their unique optical properties (i.e. strong light absorption and scattering ability within the visible and near-infrared range) as well as huge applications in sensing and biomedical science $[1,2]$. This unique ability shown by the MNPs is originated from the electromagnetic wave (EM) driven collective electron oscillations, the so called localized surface plasmons (LSPs). When frequency of the plasmon oscillation matches with the frequency of the external EM wave (light wave or evanescent wave associated with electron beam) a resonance occur which is known as localized surface plasmon resonance (LSPR). Under resonant excitation condition a huge amount of field enhancement occur around the surface of the nanostructure which is often confined spatially on sub-diffraction limited length scale of 10-50 nm and varies strongly with the morphology and composition of NPs. These highly enhanced EM field has huge applications in various field such as wave-guiding, surface-enhanced Raman scattering (SERS), sensing, photovoltaics, catalysis, and medical diagnostics. However in order to developed different applications proper understanding of the enhanced EM field around the nanostructure is of utmost importance. Most optical spectroscopic methods (such as UVVIS spectroscopy, dark field microscopy (DFM) will not be able to provide this direct correlation for nanostructured metals. The sampling volume of these techniques is much larger than the local variations in surface geometry and the measured response will be an average over the area sampled. As a result the local field enhancement around the MNPs at single particle level can't be probed accurately by traditional optical probe based techniques due to their limitations in terms of diffraction limit. Unlike photons, electron beam based spectroscopy [3, 4] such cathodoluminescence (CL) in a scanning electron microscope (SEM) [5, 6] or electron energy loss spectroscopy (EELS) [7] in transmission electron microscope (TEM) has become an excellent powerful tool for studying accurately nanoscale optical phenomena even sub nanometer resolution information in the spatial domain. Luminescence induced by electrons as excitation source (CL) has

*Electronic address: arpanmaiti25@gmail.com many advantages over luminescence based on photons as excitation source. An electron or a beam of electrons moving in vacuum can act as an evanescent source of super continuum light. The electron beam can supply all possible wave vectors required for plasmon excitation near the flat band region of the dispersion curve which can not be easily realized via optical excitation [3]. CL from metallic nanostructures relies upon the fact that energy of incident electrons is coupled to the plasmon modes of the NPs and these in turn decay into radiative photons that can be collected into far-field. As the coupling of the evanescent wave with the plasmonic eigen modes of the metal nanoparticle under investigation being highly dependent on the position of the e-beam impact, by raster scanning of the e-beam over a metal nanoparticle, one can not only captures the high resolution spectroscopic information but also record maps of spatial variation of light emission, rather the EM local density of states (EMLDOS) [3] with high spatial resolution (10-50 nm range). Thus CL technique in electron microscope is regarded as a direct probe of resonant modes of plasmonic nanostructures providing a direct way to map the local electric fields.

Among metallic nanostructure of different shape, elongated structures of $\mathrm{Au}$ such as $\mathrm{Au}$ nanorods (NRs) are especially interesting because a solid NR of gold can be thought of as a simplest kind of plasmonic waveguide which allows directional control of light. Moreover such structures show strong antenna effects as well as can support spectrally well separated multipolar LSPR modes [5]. This has versatile applications in biomedical technologies, plasmon-enhanced spectroscopies, and optical and optoelectronic devices. Here we have studied the LSPR induced optical properties of an isolated single sharp tipped Au NR (length $640 \mathrm{~nm}$ and width $200 \mathrm{~nm}$ ) using CL spectroscopy and imaging technique.

\section{EXPERIMENTAL DETAILS AND METHODS}

\section{A. Material Synthesis:}

In the present work sharp tipped gold nanorods are synthesized by a seed mediated growth method which is a small variant of the protocol used by $\mathrm{Wu}$ et al. [8]. Before doing any microscopy and CL study, the colloidal $\mathrm{Au}$ solution was centrifuged at $6000 \mathrm{rpm}$ for $15 \mathrm{~min}$, twice, and redispersed in a suitable amount of water. Fi-

\section{American Journal of Physical Sciences and Applications US:ISSN 2644-0792 website:http://thesmartsociety.org/ajpsa/}



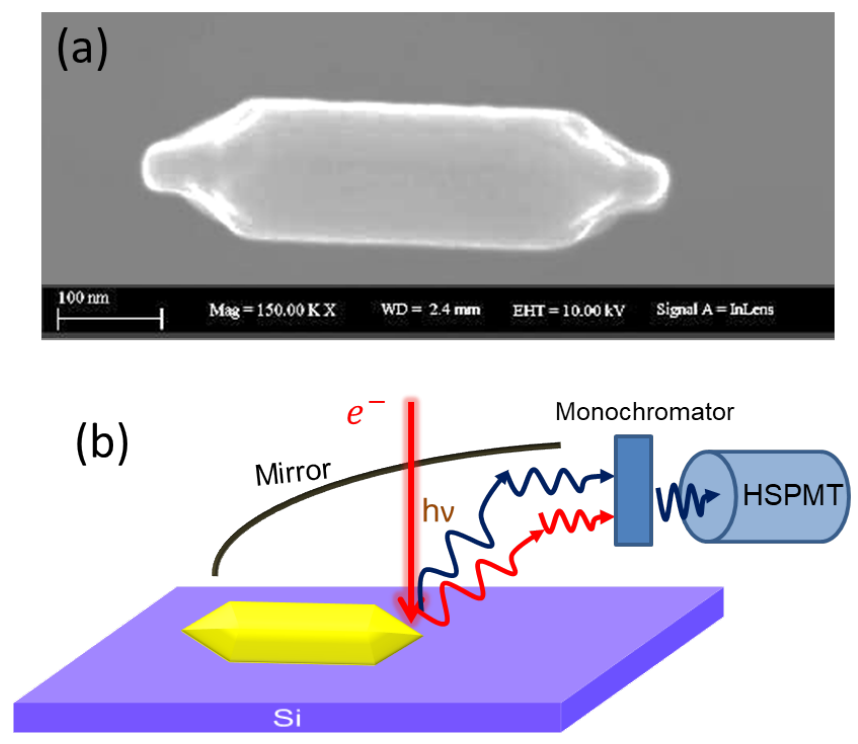

FIG. 1: (a) Secondary Electron (SE) image of the as synthesized nanorod (b) Schematic diagram of CL-SEM setup

nally, a few microliters of the diluted solution were drop coated on a cleaned Si (100) substrate and then loaded into the SEM chamber after sufficient drying in ambient condition. This synthetic approach produces a mixture of nanorods, nanospheres, triangular prisms of different sizes scattered throughout the whole drop casted region. Among the randomly distributed nanoparticles, an isolated single sharp tipped $\mathrm{Au}$ nano-rod (length $640 \mathrm{~nm}$ and width $200 \mathrm{~nm}$ ) was identified in SEM for CL measurements.

\section{B. Cathodoluminescence Measurements}

CL spectroscopy and imaging on an sharp tipped individual isolated Au nanorod are performed in a ZEISS SUPRA 40 SEM extended with the Gatan MonoCL3 CL optical detection system [9]. A schematic diagram of the CL-SEM is shown in Figure 1 (b). During the interaction of e-beam with the sample various signal is generated along with the light or photon signal. Here we are collecting the emitted light using Gatan MonoCL3 CL optical detection system. During CL experiment, the retractable paraboloidal light collection mirror is inserted manually to a position directly beneath the pole piece of SEM so that the electron beam passes through the 1 mm diameter hole of the mirror. MonoCL3 system uses a retractable paraboloidal light collection mirror that collects emitted light of the full 2 of the upper half sphere and collimates it through a hollow aluminum tube to a $300 \mathrm{~mm}$ CzernyTurner type optical monochromator, and finally the signal is fed to a high sensitivity photomultiplier tube (HSPMT). Monochromatic and panchromatic are the two mode of operation in CL-SEM system through which emitted light can be collected from the sample surface. In monochromatic mode, one can collect the spectra as a spot mode by positioning the focussed e-beam on a desired location, as well as can map the spatial variation of the photon emission at a particular wavelength by raster scanning the e-beam over the nanoparticle. In the panchromatic mode of imaging, emitted light skips the monochromator is directly carried to the HSPMT. In the present study, all the spectra have been taken in the wavelength range of 500-700 nm, averaged for each e-beam position and corrected from the substrate background. The spectral step size of $4 \mathrm{~nm}$ and a dwell time of $0.25 \mathrm{~s}$ with a band pass of $11 \mathrm{~nm}$ were maintained throughout the experiment.

\section{RESULTS DISCUSSION}

Figure 1(a) shows the high resolution SEM image of the as synthesized Au nanorod of aspect ratio (AR) 3.2 (length $640 \mathrm{~nm}$ and width $200 \mathrm{~nm}$ ). From the SE image it is very much clear that unlike conventional nanorods of cylindrical ends, our Au NR consists of very sharp tips at the two ends of the rod. So here we are interested to study the plasmon assisted photon emission from this sharp tipped Au nanorod both in spectral and spatial domain. In order to understand the plasmonic behaviour of this substrate supported Au nanorod, we have performed site specific spectroscopy and imaging using CLSEM technique. Figure 2 (a), shows experimentally obtained CL spectra acquired from different beam injection positions (marked as colored dots on the SEM images in the insets of Figure 2a) of the NR. When we excite at point 1 , i.e. right tip of the nanorod, we obtained a peak around $605 \mathrm{~nm}$, whereas the excitation from the left tip of the rod (marked as point 3) gives a peak around 580 $\mathrm{nm}$ with highest intensity. Now when the probe of the ebeam move towards point 2, 4 and 5 we obtained a peak around $530 \mathrm{~nm}$ with almost 2.5 fold decrease in intensity compare to the highest intensity obtained at excitation at tip excitation at point 3 . When the probe of the e-beam (which acts as super-continuum source of all wavelength) excite different points of the Au Nanorod, it basically excite different LSPR modes which subsequently decays to photon and we are collecting these photons arising from the Au NR using CL spectroscopy.

Now in order to understand how the light is emitted from the NRs, in Figure 3(b-d) we have also mapped the monochromatic photon maps at wavelengths 530, 580, and $610 \mathrm{~nm}$ respectively. A change in spatial distribution of the luminescence intensity at different resonant wavelength is clearly observed over a length scale much much smaller than the wavelength of the emitted light. Here the spatial variation of photon emission is caused when the field produced by the e-beam couples strongly to the EM eigen modes of $\mathrm{Au}$ NR. The monochromatic CL image at $530 \mathrm{~nm}$ (figure $3 \mathrm{~b}$ ) shows that the photon is emitted from the tip as well as the edge of the NR. On contrary to this monochromatic CL images at 580 


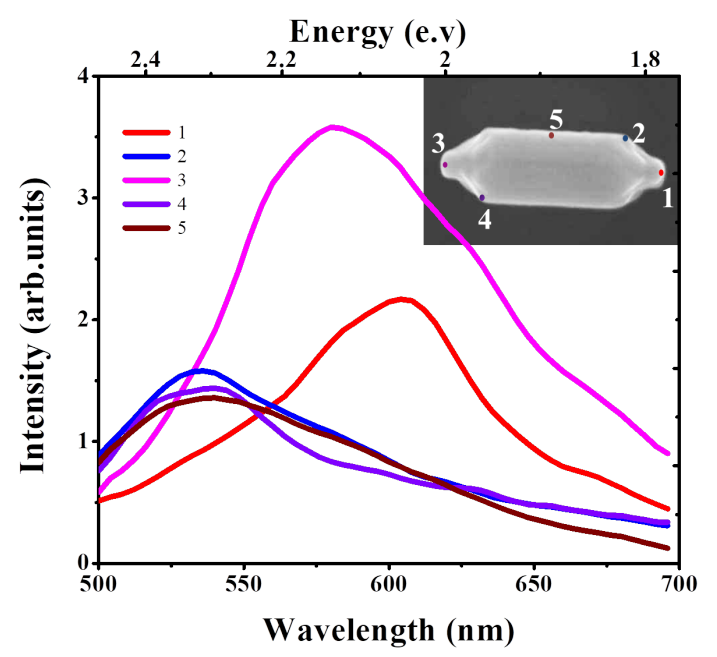

FIG. 2: Experimental CL spectra taken at different beam injection positions (indicated on the marked inset SEM image) on a Au nanorod of aspect ratio (AR) 3.2 (length $640 \mathrm{~nm}$ and width $200 \mathrm{~nm}$ ).

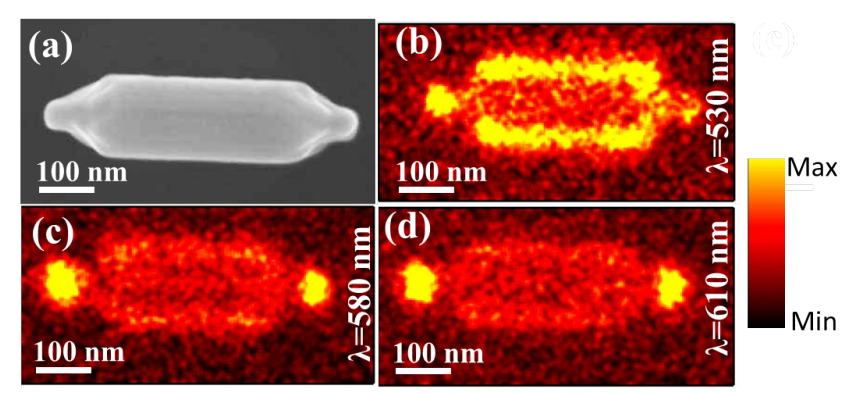

FIG. 3: (a)SEM image of the nanorod and corresponding monochromatic CL images taken (b) $530 \mathrm{~nm}$ (c) $580 \mathrm{~nm}$ (f) $610 \mathrm{~nm}$. Scale bar is $100 \mathrm{~nm}$ in all cases.

nm (Figure 3c) and $610 \mathrm{~nm}$ (Figure 3c) show maximum photon is emitted from the tip of the rod compare to the edge.

Another interesting point to note from all these monochoromatic CL images is that luminescence from the left tip (marked as point 1) is always higher intensity than the right tip (marked as point 3), which is also consistent with our measured spectra. As point 1 and 3 are two identical points of the rod so one should expect a similar spectral and spatial response from these two points. However the discrepancy arises in the present study might be arises from the sharpness of the two tips that can strongly effect the spectral position and width of the plasmon bands [10]. In the present study the observed $530 \mathrm{~nm}$ peak is the well-known transverse mode of the rod. As the NR sitting on a Si substrate (refractive index 4) so the major longitudinal dipole peak should be expected at a much red shifted wavelength than $610 \mathrm{~nm}$. So the observed peak at 580 and $610 \mathrm{~nm}$ are basically higher order mode or mixtures of the transverse dipolar mode and the horizontal quadrupolar mode [5]. In order to understand the exact origin of the different peaks numerical analysis is needed which is underway.

\section{CONCLUSION}

In summary, using CL-SEM facility we have investigated the LSPR induced photon emission from an isolated sharp tipped single Au nanorod of aspect ratio (AR) 3.2 (length $640 \mathrm{~nm}$ and width $200 \mathrm{~nm}$ ). We have also observed three different resonant peaks and also mapped the spatial variation of light emission at these resonant wavelengths. The experimental results presented here would enrich the understanding of light matter interaction at nanometer scale and may have strong implications in novel applications, such as in surface-enhanced Raman scattering, catalysis, sensing, and imaging.

\section{Acknowledgments}

The author would like to thank Prof. Tapas Kumar Chini and Dr. Achyut Maity for their support during experiment. The author is grateful to the Department of Atomic Energy (DAE), Government of India for the financial support.
[1] S.A. Maier, Plasmonics: fundamentals and applications, Springer, 1161 (2007).

[2] M. Kim, J. H. Lee, and J. M. Nam, Plasmonic photothermal nanoparticles for biomedical applications, Adv. Sci 6, 1900471 (2019)

[3] F. J. García De Abajo, Optical Excitations in Electron Microscopy, Rev. mod. Phys. 82, 209 (2010)

[4] M. Kociak, and O. Stéphan, Mapping Plasmons at the Nanometer Scale in an Electron Microscope, Chem. Soc. Rev.43, 3865 (2014)

[5] P. Das and T.K. Chini, Substrate Induced Symmetry Breaking in Penta-Twinned Gold Nanorod Probed by
Free Electron Impact, J. Phys. Chem. C, 118, 26284 (2014)

[6] A. Maiti, A. Maity, B. Satpati, N. Large, and T.K. Chini, Efficient excitation of higher order modes in the plasmonic response of individual concave gold nanocubes, J. Phys. Chem. C, 121, 731 (2017)

[7] G. Li, C. Cherqui, Y. Wu, N.W. Bigelow, P.D. Simmons, P.D. Rack, D. J. Masiello, and J.P. Camden, Examining Substrate-Induced Plasmon Mode Splitting and Localization in Truncated Silver Nanospheres with Electron Energy Loss Spectroscopy. J. Phys. Chem Lett. 6, 2569 (2015) 
[8] H-Y. Wu, H-C. Chu, T-J. Kuo, C-L. Kuo, and M. H. Huang, Seed-Mediated Synthesis of High Aspect Ratio Gold Nanorods with Nitric Acid, Chem. Mater., 17, 6447 (2005)

[9] P. Das and T.K. Chini, An Advanced Cathodoluminescence Facility in a High-Resolution Scanning Electron Microscope for Nanostructure Characterization, Curr.
Sci., 101, 849 (2011)

[10] E. Ringe, J. Zhang, M.R Langille, C.A Mirkin, L.D Marks, and R. P Van Duyne, Correlating the Structure and Localized Surface Plasmon Resonance of Single Silver Right Bipyramids, Nanotechnology, 23, 444005 (2012) 